\title{
Research on the measurement system of drilling while engineering parameters for intelligent drilling in coal mine
}

\author{
LIAN Jie \\ Xi' an Research Institute of China Coal Technology and Engineering Group Corp, 710077, Xi' an, Shaanxi, China
}

\begin{abstract}
In order to ensure safe and efficient mining and improve the efficiency of drilling construction, intelligent drilling technology has been studied in China. This technology is another development on the basis of automation. In addition to the automatic execution of the construction process, it also has the characteristics of intelligent perception, intelligent decision-making and intelligent correction. This technology requires engineering parameter measuring equipment to obtain the engineering parameters such as torque, WOB, inner and outer annulus pressure, rotation speed, vibration, temperature, etc. near the drill bit at the bottom of the hole in real time, so as to realize the real-time monitoring of the drilling process parameters at the bottom of the hole and the stress state of the drilling tool in the process of directional drilling, and increase the effective extraction distance of the drilling hole.
\end{abstract}

\section{Research background}

Coal is the main energy and important industrial raw materials in China. In order to ensure the safe and efficient mining of coal mines, intelligent drilling technology has been studied in China. This technology is another development based on automation. In addition to the automatic execution of the construction process, it also has the characteristics of intelligent perception, intelligent decision-making and intelligent correction. At present, intelligent drilling technology is still in its infancy. This technology also needs to tackle key problems such as intelligent perception of working conditions, intelligent recognition of coal and rock, intelligent control of drilling and intelligent design of drilling, so as to promote the upgrading of drilling technology, so as to realize the intelligent analysis of working conditions and correction of drilling strategy in the whole process of drilling construction and the whole operation line, which is conducive to the realization of closed-loop automatic control of intelligent drilling machine.

The existing downhole drilling equipment can only obtain some drilling parameters from the drilling instrument, such as feed pressure, pull-out pressure, rotary pressure, etc. These parameters can not directly reflect the drilling process parameters such as torque, WOB, inner and outer annulus pressure, rotation speed, vibration, temperature and the stress state of the drilling tool near the bit in the process of drilling. In the process of drilling construction, workers usually estimate the engineering parameters in the hole subjectively according to the values on the pressure gauge outside the hole. The measurement of downhole temperature can reflect the environment in the hole, which is of practical significance to reflect the formation conditions, protect drilling tools and measuring tools. Real time measurement of torque, WOB, rotation speed and vibration can detect the stress of the whole drill string, understand the status of the drill bit, and prevent the occurrence of accidents such as sticking. Measuring the inner and outer annulus pressure can show the wellbore pressure at any time, which is an important means to ensure drilling safety, avoid sticking and prevent hole collapse. Therefore, the above parameters have a great impact on the drilling efficiency, and as measurable and controllable parameters.

Obtaining the above parameters can not only improve the design level of LWD instruments, but also optimize the drilling technology, which is the necessary technical support for intelligent drilling equipment and efficient and rapid drilling technology in coal mines. The system is used with the intelligent drilling machine to provide reliable data to help the drilling machine effectively adjust the drilling attitude, improve the drilling efficiency and prevent accidents in the hole.

\section{Design ideas}

The MWD engineering parameter measurement system for intelligent drilling in coal mine needs to be able to observe the parameters such as torque, WOB, inner and outer annulus pressure, rotation speed, vibration, temperature in the borehole in real time, and transmit the collected data to the borehole monitor in real time through the wireline drill pipe.

The following problems need to be considered in the structural design of the equipment. Due to the particularity of the environment, there is no corresponding standard sensor for the measurement of

\footnotetext{
*Corresponding author: lianjie@cctegxian.com
} 
torque and WOB in coal mine. Therefore, it is necessary to design a special sensor to measure. From the measurement of the elastomer mechanism to the implementation of the process, full consideration should be given to the performance of the measurement parameters to meet the actual use, leaving a margin space for adjustment. The underground environment is complex and changeable, and the condition is bad, which puts forward high requirements for the structure of the equipment. In the design, it is necessary to improve the adaptability of the equipment to ensure that the measurement can reduce environmental interference factors, and meet the requirements of GB3836 and coal mine safety signs. The system will be a part of the drill string and also play a role of transmission, so it is necessary to have enough safety strength to ensure that the safety factor of the ultimate strength of the structure should be left with margin. In the drilling engineering, the requirements of drilling tools are very strict, once there is an accident, it will cause huge losses.

\section{Design scheme}

In order to adapt to the downhole drilling conditions, it is necessary to analyze the axial force, torque, internal and external extrusion pressure and bending moment of the instrument in the borehole, as well as the possible vibration, impact and temperature changes during the drilling construction, design the instrument structure, ensure that the strength of the instrument is not lower than that of the same kind of drill pipe, and meet the drilling construction requirements. As shown in Figure 1.

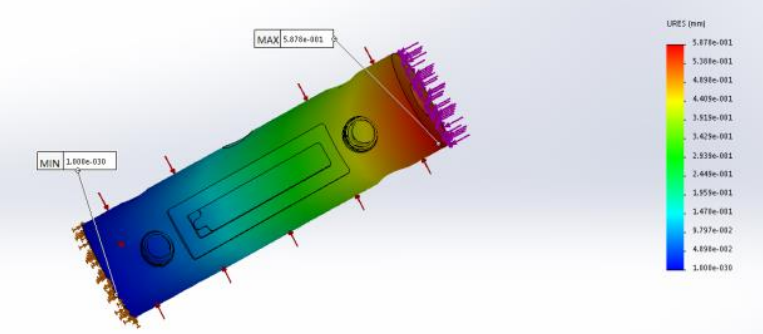

Fig. 1. Results of finite element analysis.

In order to realize the measurement function, the instrument also needs to be designed including drill collar body, control circuit, vibration sensor, communication module, drill pressure torque strain gauge, outer annulus pressure sensor, inner annulus pressure sensor, rotation speed sensor and temperature sensor, etc.

The measurement of torque, WOB and other parameters is the design difficulty of the MWD engineering parameter measurement system. In the design, resistance strain electrical method, magnetostrictive elastic method, surface acoustic wave method, angle phase difference method, meshing cylinder method, piezoelectric electrical method and other forms are usually used.

The internal and external annulus pressure is usually measured by introducing the built-in sensor or fiber
Bragg grating method. Many factors need to be fully considered in the design of the engineering parameter measurement system of drilling while mining. First, the performance of measurement parameters shall meet the actual use, with margin space reserved for adjustment; second, the safety shall be fully guaranteed, and margin shall be reserved for the safety factor of structural ultimate strength; third, the environmental impact shall be fully considered, and the structural scheme shall try to eliminate interference; Fourth, the design shall meet the requirements of GB3836, and the feasibility and cost of scheme implementation shall be considered.

The relationship between these principles is that the measurement is based on safety considerations, while the structural measurement and safety assurance should reduce the interference of the environment and not cause too much impact on the environment. The overall design should also be based on the compliance with GB3836. These aspects form the structural form of layers of buckle.

The following problems need to be considered in the circuit design of the equipment.

First of all, in order to meet the requirements of coal mine underground use, the circuit design of the equipment should conform to GB3836, through the power carrier module to realize the power / signal composite transmission in the drill pipe. The parameters such as torque, WOB, inner and outer annulus pressure, rotation speed, vibration and temperature collected by the engineering parameter measurement system shall be able to be transmitted to the orifice in real time through the cable, so the communication module shall be designed and the data shall be transmitted through the cable drill pipe. The working time of the engineering parameter measurement system should be as long as possible, which can cover the drilling construction. Therefore, the design adopts the way of orifice power supply, and the power is transmitted to the engineering parameter measurement system in the hole through the cable drill pipe.

\section{Design scheme}

At present, this technology is still blank in the field of coal mine. In the design, we need to develop supporting software, consider the design of the corresponding visual operation interface, and the operation needs to conform to the underground drilling process flow of coal mine, so that the operators can observe the engineering parameters. The research and development of the system can not only provide reliable data to help the drilling machine effectively adjust the drilling posture, so as to improve the drilling efficiency, but also effectively prevent accidents in the hole to avoid losses and protect the efficient drilling construction.

At the same time, the development trend of intelligent drilling construction is MWD + MWD + rotary guidance. It will realize a great leap from "geometry guided drilling" to "precise geological guided drilling" to "automatic rotary guided drilling" Only when the engineering parameters are acquired in real time 
while drilling can the closed-loop control of rotary steering be realized, which is a key technology to be solved in the development of intelligent drilling.

\section{References}

1. Z. Yu, Z. Lala. Development and field application of downhole engineering parameter measuring instrument. J. Mud Logging Engineering 28, 1317,22 (2017)

2. Z. Haihua, C. Haibo. Character analysis of a new device for measuring weight and torque near the bit. J. Electronic Design Engineering 19, 49-52 (2011)

3. W. Yinsheng, Y. Jinzhou, H. Laij. Development Of MWD equipment. J. Petroleum Instrument, 23, 3536(2009)

4. F. Yingjun, M. Tianshou, C. Ping. Finite element analysis of the short section of underground engineering parameter measurement. J. China Petroleum Machinery, 05, 41-44(2011)

5. M. Tianshou, C. Ping, H. Yuan. Design and manufacture of downhole engineering parameter measurement short section. J. Machinery Design \& Manufacture, 11, 28-30(2011)

6. H. Ze, X. Yuheng, G. Liang. Research on bridge and plate arrangement of strain type downhole engineering parameter measuring sensor group. J. Process Automation Instrumentation, 33, 7779(2012): 